\title{
A study on the plane figures of elementary school in the view of transformation geometry
}

\author{
Keunbae Choi, Dept. of Math. Education, Teachers College, Jeju National University, kbchoe@jejunu.ac.kr
}

Abstract. One of the main purposes of geometry learning is the classification of shapes. Thus the activity of classifying shapes from a geometric symmetry (invariant) point of view has sufficient educational significance from Klein's point of view. The concept of translation, line symmetry, and rotational symmetry is introduced as part of activities to raise spatial sense in the current elementary school mathematics curriculum. The above three transformations are basic activities in the concept of congruence. But the current mathematics curriculum tends to deal with three basic concepts and congruence regardless of whether they are related. In fact, three activities and congruent concepts for developing spatial senses was introduced in different the grade-band for each other.

In this paper, we shall classify triangles and quadrilaterals from the viewpoint of a symmetry breaking

Keywords: Line symmetry, Rotational symmetry, Group, Triangle, Quadrilateral..

Received: 14.12.2020 Accepted: 12.01.2021 $\quad$ Published: 07.02.2021

\section{INTRODUCTION}

In all geometries, Klein [1, 2, 3] connected a group of basic symmetry. The hierarchy of geometry is therefore expressed mathematically as a hierarchy of these groups and as a hierarchy of their invariant. For example, length, angle, and area are preserved with respect to the Euclidean group, while only the incident structure and cross-ratio are preserved under the most common projective transformation. The concept of parallelism preserved in affine geometry has no meaning in projective geometry. By abstracting the group of basic symmetry in geometry, the relationship between them can be reset at the group level. Since the group of affine geometry is a subgroup of projective geometry, the unchanging concept in projective geometry is a priori concept with meaning in affine geometry, but not vice versa. There are more powerful theories when the necessary symmetry is removed, but fewer concepts and theorem.

Symmetry is a very important topic in mathematics and physics that describes physical phenomena. In 2008, three physicists from Japan won the Nobel Prize in Physics for their work on symmetry breaking [4]. The symmetrical breakage they discovered is believed to have existed since the beginning of the universe. Immediately after the Big Bang, materials and antimatter were symmetrical, and at some point, the symmetry was broken, leaving only materials, which formed the current universe.

The concept of translation, line symmetry, and rotational symmetry is introduced as part of activities to raise spatial sense in the current elementary school mathematics curriculum. The above three transformations are basic activities in the concept of congruence [5, 6]. But the current mathematics curriculum tends to deal with three basic concepts and congruence regardless of whether they are related. One of the main purposes of shape learning is the classification of shapes. Therefore, the activity of classifying shapes from the perspective of symmetry has sufficient educational significance from Klein's point of view [1].

\section{Backgrounds}

The Erlangen program, published in 1872 by Felix Klein as "Comparative Review of Recent Geometry Studies," proposes a research methodology that characterizes geometry based on group theory and projective geometry [1]. Around 1872, non-Euclidean geometry [5] appeared, but there was no way to determine its relationship and class. Klein's method was fundamentally innovative in three ways: First, projective geometry was emphasized by him as an integrated frame of all other geometries considered. In particular, Euclidean geometry is more restrictive than Affine geometry, and in turn, affine geometry is more restrictive than projective geometry. Second, Klein suggested that group theory, a branch of mathematics that uses algebraic methods to abstract the concept of symmetry, was the most useful way to organize geometric knowledge; at that time, group theory was already introduced into equation theory in the form of Galois theory. Third, Klein further clarified the idea that there are appropriate concepts for each geometric language. For example, projective geometry is suitable for dealing with conic sections, but 
it is not suitable for dealing with the concept of a circle or angle, which is not invariant in the projective transformation.

The way the languages of geometry are reassembled can be explained in such a way that the subgroups of symmetric groups are interrelated.

\section{Viewing plane figures in the perspective of symmetries}

In the current Korean mathematics curriculum, activities related to the transformation of plane figures are introduced only in the first semester of the fourth grade [7]. In the case of the United States (Harcourt Math [8]), activities to develop spatial sense are introduced over all grades (levels), and the content is also wider and more diverse than in our cases [8,9]. For example, the activities of tessellation, fractal, and symmetric line drawing. In particular, in the case of rotational transformation, unlike ours, which only deals with $120^{\circ}, 240^{\circ}$, and $360^{\circ}$, as shown in Figure 1, the viewpoint of transformation is handled more generally.

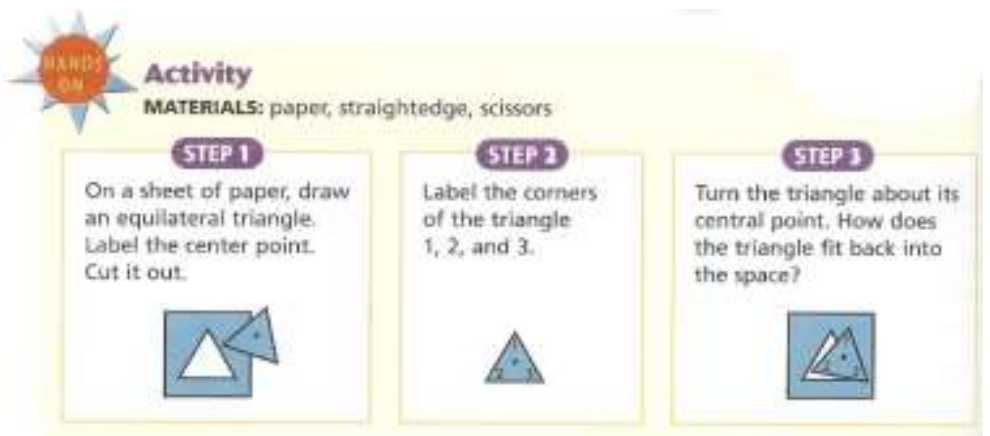

Figure 1. Activities of rotational symmetry [8]

In addition, the concept of congruence and linear symmetry is introduced in the United States from the lower grades, while in our case, the perspective of transformation is ignored in the second semester of the fifth grade, and in our case, it is introduced from the perspective of "two overlapping shapes," and the main activities are what the corresponding points, the corresponding lines of the two shapes are. That is, there is no perspective on transformation-slide, flip, and turn-that occurs while one shape moves and overlaps with another. It focuses only on overlapping results.

Overall, our textbooks lack spatial sense activities in terms of transformation (symmetrical) than in the United States. Therefore, it is necessary to classify the planar shapes in terms of symmetry.

\subsection{Circle}

Looking at a circle from the perspective of symmetry, it is a figure with infinite number of line and rotational symmetry. More mathematically, the symmetry group of a circle is the orthogonal group $O(2)$ :

$\mathrm{O}(2)=\left\{A \in \mathrm{GL}(2): A^{T} A=I=A A^{T}\right\}$

Where GL(2) is the general linear group of $2 \times 2$ real matrices.

3.2. Triangle

Elementary mathematics textbooks of current curriculum in Korea [6] introduce triangles from two different perspectives. One is classified by 'angle': right triangle, acute triangle, and obtuse triangle, and the other is classified by 'side': equilateral triangle and isosceles triangle. Although most of the triangles are scalene, they are not handled.

As part of developing a sense of space, let's introduce an activity that classifies triangles in terms of symmetry (see, Figure 2). First, it is a rotation about a straight line perpendicular to the plane containing this equilateral triangle through the center of gravity 0 . There are three rotations with rotation angles of $0^{0}, 120^{\circ}, 240^{\circ}$, respectively. In addition, there are three $180^{\circ}$ rotations of the axis of symmetry (1), (2), and (3).

This set of six rotations forms a group. In general, this group is called the (dihedral group) of order 6 and is often referred to as $D_{3}$. In other words,

$$
D_{3}=\left\{1, R_{120}, R_{240}, r_{1}, r_{2}, r_{3}\right\}
$$




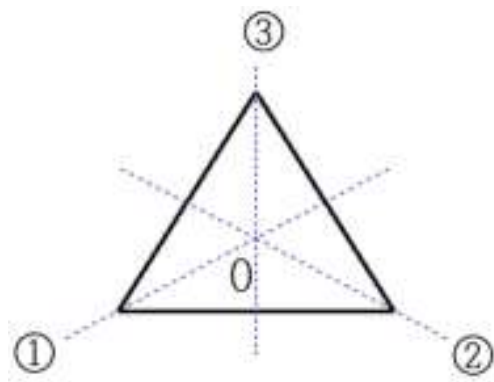

Figure 2. Symmetries of equilateral triangle

The multiplication table for this group is as follows.

Table 1: Multiplication table of $D_{3}$

\begin{tabular}{c|cccccc}
$\cdot$ & 1 & $R_{120}$ & $R_{240}$ & $r_{1}$ & $r_{2}$ & $r_{3}$ \\
\hline 1 & 1 & $R_{120}$ & $R_{240}$ & $r_{1}$ & $r_{2}$ & $r_{3}$ \\
$R_{120}$ & $R_{120}$ & $R_{240}$ & 1 & $r_{2}$ & $r_{3}$ & $r_{1}$ \\
$R_{240}$ & $R_{240}$ & 1 & $R_{120}$ & $r_{3}$ & $r_{1}$ & $r_{2}$ \\
$r_{1}$ & $r_{1}$ & $r_{3}$ & $r_{2}$ & 1 & $R_{240}$ & $R_{120}$ \\
$r_{2}$ & $r_{2}$ & $r_{1}$ & $r_{3}$ & $R_{120}$ & 1 & $R_{240}$ \\
$r_{3}$ & $r_{3}$ & $r_{2}$ & $r_{1}$ & $R_{240}$ & $R_{120}$ & 1
\end{tabular}

Therefore, it can be seen that in the case of the subgroup of order 3: $A=\left\{1, R_{120}, R_{240}\right\}$, in the case of order 2: $\left\{1, r_{1}\right\},\left\{1, r_{2}\right\}$, and $\left\{1, r_{3}\right\}$. The subgroup $A$ still maintains an equilateral triangle despite losing 3 symmetries at. However, in the case of order 2 , it can be seen that the 4 symmetries at are lost, resulting in an isosceles triangle. In addition, the scalene triangle is obtained through the process of symmetry breaking [11] of the isosceles triangle (see, Figure 3).

Equilateral Triangle

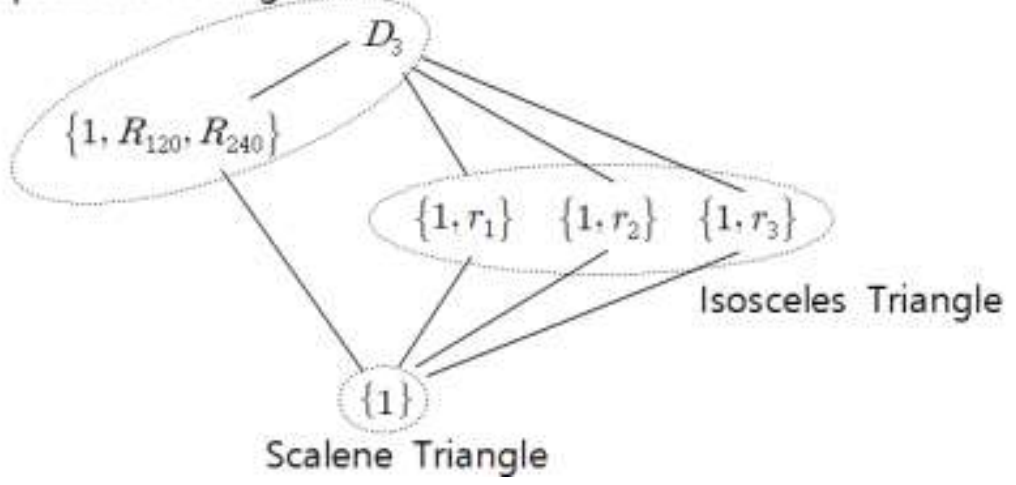

Figure 3. Classifying triangles in view of symmetry

An equilateral triangle, an isosceles triangle, and an equilateral triangle are created through the process of symmetrical breaking as shown in Figure 4.
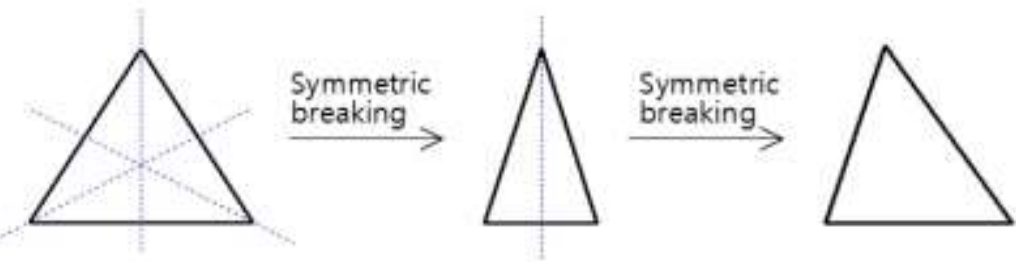

Figure 4. Formation of equilateral triangle, isosceles triangle, scalene triangle

\section{Quadrilateral}

Looking at quadrilateral in the perspective of symmetry, the quadrilateral has many symmetries. In fact, it has 4 rotational symmetries and 4 linear symmetries. Let's take a more logical look at the various quadrilateral that emerge from the symmetry breaking of the square. First, it is a rotational symmetry with a straight line perpendicular to the plane containing this square, passing through the center of gravity 0 , as its axis, and there are four rotations $1, R_{90}, R_{180}, R_{270}$ with rotation angles of $0^{0}, 90^{\circ}, 180^{\circ}$, 
$270^{\circ}$ respectively. In addition, there are 4 line symmetry $H, V, D_{1}, D_{2}$ with the axis of symmetry (1), (2), (3) and (4) as axes (See, Figure 5).

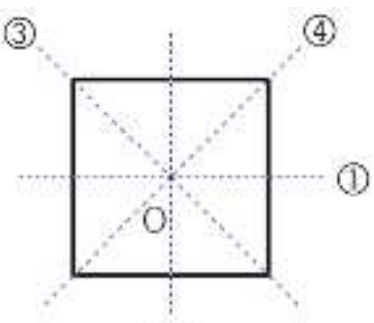

(2)

Figure 5. Symmetries of quadrilateral

Such a set of eight symmetries constitutes a group. This group is commonly referred to as the dihedral group of order 8 and is often referred to as $D_{4}$. Namely,

$D_{4}=\left\{1, R_{90}, R_{180}, R_{270}, H, V, D_{1}, D_{2}\right\}$

And the multiplication table for this group is as follows [10].

Table 2: Multiplication table of $D_{4}$

\begin{tabular}{c|cccccccc}
$\cdot$ & 1 & $R_{90}$ & $R_{180}$ & $R_{270}$ & $H$ & $V$ & $D_{1}$ & $D_{2}$ \\
\hline 1 & 1 & $R_{90}$ & $R_{180}$ & $R_{270}$ & $H$ & $V$ & $D_{1}$ & $D_{2}$ \\
$R_{90}$ & $R_{90}$ & $R_{180}$ & $R_{270}$ & 1 & $D_{2}$ & $D_{1}$ & $H$ & $V$ \\
$R_{180}$ & $R_{180}$ & $R_{270}$ & 1 & $R_{90}$ & $V$ & $H$ & $D_{2}$ & $D_{1}$ \\
$R_{270}$ & $R_{270}$ & 1 & $R_{90}$ & $R_{180}$ & $D_{1}$ & $D_{2}$ & $V$ & $H$ \\
$H$ & $H$ & $D_{1}$ & $V$ & $D_{2}$ & 1 & $R_{180}$ & $R_{90}$ & $R_{270}$ \\
$V$ & $V$ & $D_{2}$ & $H$ & $D_{1}$ & $R_{180}$ & 1 & $R_{270}$ & $R_{90}$ \\
$D_{1}$ & $D_{1}$ & $V$ & $D_{2}$ & $H$ & $R_{270}$ & $R_{90}$ & 1 & $R_{180}$ \\
$D_{2}$ & $D_{2}$ & $H$ & $D_{1}$ & $V$ & $R_{90}$ & $R_{270}$ & $R_{180}$ & 1
\end{tabular}

As shown in Figure 7, from the subgroup lattice diagram of $D_{4}$, all quadrilateral introduced in elementary school mathematics can be viewed from the perspective of symmetry breaking.

- The quadrilateral corresponding to the $D_{4}$ and cyclic group $\left\{1, R_{90}, R_{180}, R_{270}\right\}$ is a square.

- The symmetric group $\left\{1, R_{180}, V, H\right\}$ of rectangular is commonly called the Klein 4-group. We note that the group of order 4 is isomorphic to a cyclic group of order 4 or the Klein 4-group. In fact, the symmetric group $\left\{1, R_{180}, D_{1}, D_{2}\right\}$ of a rhombus is isomorphic to the Klein 4-group, and the cyclic group $\left\{1, R_{90}, R_{180}, R_{270}\right\}$ created by the rotational symmetry of the square is not isomorphic to the Klein 4group. According to Piaget [12], the transformations associated with the thinking of students of the formal operational stage actually create Klein 4-group (Figure 6).

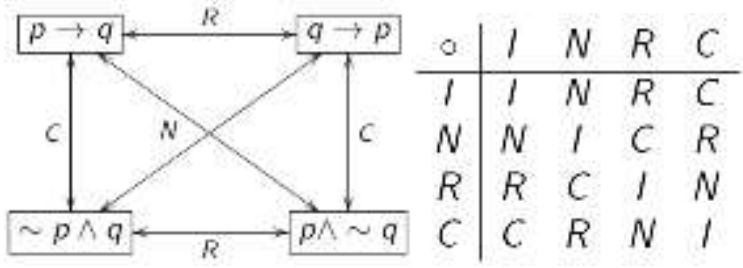

Figure 6. Piaget's INRC group 


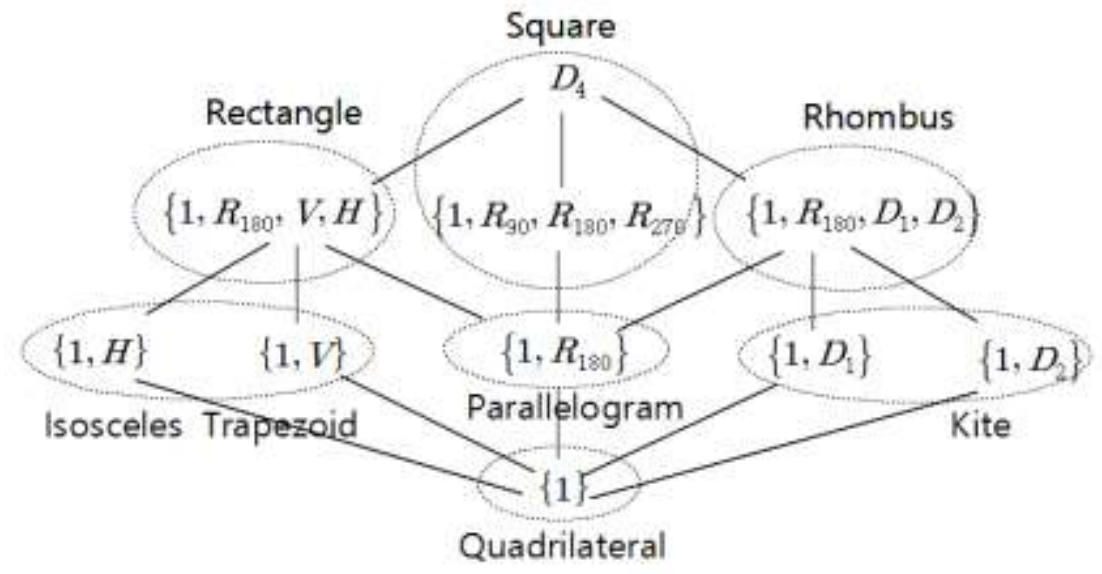

Figure 7. Classifying quadrilateral in view of symmetry

\section{Conclusion and Discussion}

Thinking from the standpoint of elementary mathematics education that the present universe was formed through the symmetry breaking is somewhat lacking in specifics but can be a very interesting fact for students. Out of the Plato essentialist concept explanation, the method of explanation taking into account the change of time can also interest students in math learning. For example, the following storytelling about the birth of a rectangle can attract students' interest: In the beginning, a circle with the most perfect symmetry lost its symmetry and became a square, but the square also lost its symmetry and became a rectangle.

Meanwhile, Euclidean group $\mathrm{E}(n)$ associated with the congruent transformation is in fact semi-direct product of orthogonal (rotation and linear symmetry) groups with translations. This represents the group action, and the group action is the transformation activity. Therefore, it is also considered in the educational perspective that sliding, flipping, and turning, which are the causes of congruent transformation, are experienced in the early stage in the view that the flow of transformation geometry is emphasized under the historical change - proof geometry of mathematics.

Also, according to Piaget, the transformations associated with the thinking of students of the formal operational stage actually create Klein 4-group, the symmetric group of rectangular. This shows the importance of the group from a psychological point of view. In other words, the group is a transformation and a psychological activity.

\section{Acknowledgements}

This work was supported by the Ministry of Education of the Republic of Korea and the National Research Foundation of Korea (NRF-2019S1A5C2A04083374).

\section{References}

[1] Klein, F. (1872) A comparative review of recent researches in geometry: Complete English Translation is in here https//:arxiv.org/abs/0807.3161.

[2] Thomas Hawkins. (1984). The Erlangen program of Felix Klein: Reflections on Its Place in the History of Mathematics. Historia Mathematica 11 (4), 442-470.

[3] Information on https://en.wikipedia.org/wiki/Erlangen program.

[4] The Novel Foundation. (2008). The Novel Prize in Physics 2008. Nobelprize.org. Retrieved Jan. 15. 2008.

[5] Ryan, P. J. (1986). Euclidean and non-Euclidean geometry; an analytic approach. New York: Cambridge University Press.

[6] Anton, H. (1981). Elementary Linear Algebra. $3^{\text {rd }}$ Ed. John Wiley \& Sons, New York, p. 240.

[7] Ministry of Education. (2018). Mathematics; Grade 1- Grade 6. Chunjae Inc, Seoul.

[8] Evan, M. M et al. (2002). Harcourt Math (Math-Grade 4). Harcourt. Inc.

[9] Choi, K. B and H. G. Kim (2005). A comparative study on elementary school mathematics textbooks in Korea ( $7^{\text {th }}$ curriculums) and America (Harcourt Math); focused on the area of geometry. J. Korea Soc. Ed. Ser. A: The Mathematical Education 44(2), 179-200.

[10] Gallian, J. A. (1998). Contemporary Abstract Algebra. $4^{\text {th }}$ Ed. Houghton Mifflin Company, Boston, pp. 29-34.

[11] Delanda, M. (2002). Intensive Science and Virtual Philosophy. Bloomsbury, New York, chapter 1.

[12] Ascher, E. (1984). The Case of Piaget's Group INRC. Journal of Mathematical Psychology 28(3), 282316. 
